\title{
Advances in Turbulence, Heat and Mass Transfer Preface
}

\author{
Kemo Hanjalić ${ }^{1}$ • Carlo Massimo Casciola $^{2}$ - Epaminondas Mastorakos ${ }^{3}$
}

Published online: 16 October 2019

(C) Springer Nature B.V. 2019

Understanding and predicting convective heat and mass transfer, which are driven or controlled by turbulence and which feed back into the flow dynamics through multi-facetted interactions, pose ever more new challenges in various branches of engineering and the environment. New discoveries revealed by advanced numerical simulations and laser-based laboratory diagnostics, especially in reacting single- and multi-phase fluid flows, are still awaiting full understanding of the physical phenomena involved, especially of the role of turbulence, its control and manipulation. This Issue of Flow, Turbulence and Combustion aspires to highlight recent progress and to provide an archival record of some of the current trends and achievements in this research field.

This Issue contains 12 articles, selected from 135 papers presented at the 9th International Symposium on Turbulence, Heat and Mass Transfer, held in Rio de Janeiro, Brazil, in the period 10-13 July, 2018. This triennial event, initiated in 1994 under the auspices of ICHMT (International Centre for Heat and Mass Transfer), continues to provide a forum for scientists and engineers from academia and industry to present and discussing advances in research on the role turbulence in heat and mass transfer. Every paper in this Issue was subjected to the same rigorous review process as that applied to all $\mathrm{FTaC}$ submissions.

The first six articles deal with turbulent combustion. An up-to-date overview of trends and developments in modelling and experiments is given in the first article, complemented by papers on new experimentally derived insights into the physics of flames subjected to elevated

Kemo Hanjalić

k.hanjalic@tudelft.nl

Carlo Massimo Casciola

carlomassimo.casciola@uniroma1.it

Epaminondas Mastorakos

em257@eng.cam.ac.uk

1 Transport Phenomena, TU Delft / Faculty Applied Sciences, Building 58, Van der Maasweg 9, 2629 HZ Delft, The Netherlands

2 Department of Mechanical and Aerospace Engineering, Sapienza University of Rome, Via Eudossiana 18, 00164 Rome, Italy

3 Engineering Department, University of Cambridge, Trumpington Street, Cambridge CB2 1PZ, UK 
pressure and to strong swirl with vortex breakdown and distinct helical structures. These are followed by papers reporting numerical simulations of supersonic combustion, of propagation of spherical waves into fluid droplet mists, and of RANS-PDF modelling of an air-methane non-premixed flame using a simplified chemistry model. The second group of articles cover a variety of separate topics, starting with an overview of turbulence and thermal structures in the upper ocean educed from eddy-resolving simulations. The articles that follow report experiments of gas-liquid flow through an inclined T-junction, numerical simulations of pipe flow subjected to radial (orthogonal-mode) rotation and synthetic-jet control of separated flows. The Issue closes with papers reporting some unusual and intriguing computer-modelling studies of people-crowd behaviour during the dispersion of incidentally released heavy gas, and on water-droplet erosion of a compressor-cascade blade.

The editors wish to thank all authors and reviewers for their efforts towards making this Issue a valuable contribution to the archival literature on the role of turbulence in heat and mass transfer. 